\title{
FISH Karyotype and GISH Meiotic Pairing Analyses of a Stable Intergeneric Hybrid xBrassicoraphanus Line BB\#5
}

\author{
Hadassah Roa Belandres ${ }^{1}$, Nomar Espinosa Waminal ${ }^{1,2,3,4}$, Yoon-Jung Hwang ${ }^{1}$, Beom-Seok Park ${ }^{5}$, \\ Soo-Seong Lee ${ }^{6}$, Jin Hoe Huh ${ }^{2,3,4}$, and Hyun Hee Kim' \\ ${ }^{1}$ Plant Biotechnology Institute, Department of Life Science, Sahmyook University, Seoul 139-742, Korea \\ ${ }^{2}$ Department of Plant Science, Seoul National University, Seoul 151-921, Korea \\ ${ }^{3}$ Plant Genomics and Breeding Institute, Seoul Natioanl University, Seoul 151-921, Korea \\ ${ }^{4}$ Research Institute for Agriculture and Life Sciences, Seoul National University, Seoul 151-921, Korea \\ ${ }^{5}$ Agricultural Genome Center, National Academy of Agricultural Science, Rural Development Administration, Suwon 441-707, Korea \\ ${ }^{6}$ BioBreeding Institute, Ansung 456-756, Korea
}

\begin{abstract}
Brassicoraphanus} \mathrm{line} \mathrm{BB \# 5,} \mathrm{a} \mathrm{new} \mathrm{synthetic} \mathrm{intergeneric} \mathrm{hybrid} \mathrm{between} \mathrm{Brassica} \mathrm{rapa} \mathrm{L.} \mathrm{ssp.} \mathrm{pekinensis} \mathrm{and} \mathrm{Raphanus}$ sativus L. var. rafiphera induced by $\mathrm{N}$-methyl-N-nitroso-urethane mutagenesis in microspore culture, shows high seed fertility and morphological uniformity. Dual-color fluorescence in situ hybridization (FISH) using 5S and 45S rDNA probes and genomic in situ hybridization (GISH) using B. rapa genomic DNA probe were carried out to analyze the chromosome composition and the meiosis pairing pattern compared to its parental lines. The somatic chromosome complement is $2 \mathrm{n}=38$, which consists of 17 metacentric and two submetacentric chromosomes with lengths of 2.18 to $5.01 \mu \mathrm{m}$. FISH karyotype analysis showed five and eight pairs of $5 \mathrm{~S}$ and 45S rDNA loci. GISH meiosis pairing analysis showed that 19 complete bivalents were most frequent and accounted for $42 \%$ of the 100 pollen mother cells examined. Based on chromosome number, size, morphology, rDNA distribution, and meiosis pairing pattern, both parental genomes of B. rapa and $R$. sativus appear to exist in xBrassicoraphanus line BB\#5, demonstrating its genome integrity. Such stable chromosome constitutions and meiotic pairing patterns in somatic and meiotic cells are very rare in natural and synthetic intergeneric hybrids. Chromosomal studies and genetic and phenotypic changes in allopolyploids are discussed. The results presented herein will be useful for further genomic study of xBrassicoraphanus lines and their improvement as promising new breeding varieties.
\end{abstract}

Additional key words: cytogenetic study, intergeneric allotetraploid BB\#5

\section{Introduction}

Brassica and Raphanus species have been cultivated worldwide as important vegetables, fodders, and sources of functional compounds. Owing to their usefulness, many studies have been carried out to investigate breeding of these species (McNaughton, 1979; Namai et al., 1980; Olsson and Ellerstrom, 1980; Prakash et al., 2009).

Synthetic hybrid species between Brassica and Raphanus, $\mathrm{xBrassicoraphanus} \mathrm{or} \mathrm{xRaphanobrassica}$, have been developed and studied by many researchers using morphological and cytological methods (Dolstra, 1982; Kato and Tokumasu, 1983; Tokumasu, 1976). In Korea, Lee et al. $(1999,2002)$ developed a new intergeneric allotetraploid, xBrassicoraphanus, by a cross between B. rapa L. ssp. pekinensis and $R$. sativus $\mathrm{L}$ and named it 'Baemoochae'. Initially, this new synthetic hybrid was so unstable that low seed fertility and poor uniformity were continued through generations. However, a microspore mutagenesis using $\mathrm{N}$-nitroso $\mathrm{N}$-methyl urethane (NMU) created stabilized progenies from this unstable material (Lee et al., 2011). They also developed another intergeneric allotetraploid xBrassicoraphanus between Korean

\footnotetext{
*Comesponding author: kimhh@syu.ac.kr
}

※ Received 5 September 2014; Revised 17 September 2014; Accepted 17 September 2014. This study was supported by a grant from the Next-Generation BioGreen21 Program (No. PJ009093), Rural Development Administration, Korea.

(C) 2015 Korean Society for Horticultural Science 
land races of B. rapa L. ssp. pekinensis and R. sativus $\mathrm{L}$. var. rafiphera and the F1 hybrid was crossed again with a mutagen-induced stabilized line. Using the same microspore mutation technique, another stable line of xBrassicoraphanus showing quite high seed fertility and morphological uniformity was obtained, which was named BB\#5.

Chromosome-based cytogenetic studies using conventional staining method have been applied to Brassica species since 1920 to investigate chromosome composition and genome structure. Chromosome characterization and genome identification have advanced significantly with the development of molecular cytogenetics through fluorescence in situ hybridization (FISH) and genomic in situ hybridization (GISH) techniques (Capdeville et al., 2008; Fukui, 2005; Hwang et al., 2010, 2012; Levsky and Singer, 2003; Park et al., 2010). Indeed, these methods have been used to investigate genome structure and inter-genomic relationship of hybrid plants (Jellen et al., 1994; Kenton et al., 1993), allopolyploid species (Cao, 2003; Devi et al., 2005; Yang et al., 1999), and recombinant breeding lines (Hwang et al., 2012; Lou et al., 2010; Vasconcelos et al., 2010).

Tandem repeat DNA sequences including $5 \mathrm{~S}$ and $45 \mathrm{~S}$ ribosomal DNAs (rDNAs) have primarily been used as cytogenetic markers in FISH (Kato et al., 2004; Lim et al., 2005, 2012). In Brassica, Maluszynska and Heslop-Harrison (1993) first reported the number of $45 S$ rDNA loci in diploids (B. rapa, $\mathrm{n}=10$, AA genome; $B$. nigra; $\mathrm{n}=8$, $\mathrm{BB}$ genome; $B$. oleracea, $\mathrm{n}=9$, CC genome,) and allo-tetraploids (B. carinata, $\mathrm{n}=17$, BBCC genome; $B$. juncea, $\mathrm{n}=18$, AABB genome; $B$. napus, $\mathrm{n}=19$, AACC genome). Genomic distributions of rDNA sites on prometaphase and metaphase chromosomes were described more precisely upon further investigations (Fukui et al., 1998; Hasterok and Muluszynska, 2000a, 2000b; Hasterok et al., 2006; Hwang et al., 2009; Kim et al., 1998; Snowdon et al., 1997). Some chromosomes of the complements were identified exactly, enabling a detailed karyotype analysis for the genus Brassica.

Lim et al. (2012) analyzed the karyotype of the somatic metaphase of the $\mathrm{xBrassicoraphanus} \mathrm{line} \mathrm{BB} \# 4$ by conventional Giemsa-staining, but no cytogenetic studies of the new synthetic intergeneric hybrid line BB\#5 have been conducted to date. Here, we carried out FISH karyotype analysis of BB\#5 using 5S and 45S rDNA probes, with reference to its parental species B. rapa and $R$. sativus. In addition, meiotic chromosome pairing patterns were analyzed and the Brassica and Raphanus genomes were discriminated by GISH method.

\section{Materials and Methods}

\section{Plant Materials}

Seeds and flower buds of the xBrassicoraphanus line BB\#5 were provided by the Bio Breeding Institute, Ansung, Korea and seeds of its parental species, Brassica. rapa L. ssp. pekinensis and Raphanus. sativus L var. rafiphera were provided by the RDA Genebank, Suwon, Korea.

Seeds were sown on moist filter paper in Petri dishes and germinated at $25^{\circ} \mathrm{C}$ for 48 hours. Approximately 2 $\mathrm{cm}$ long root tips were harvested from the germinated seeds, pretreated in $2 \mathrm{mM}$ 8-hydroxyquinoline at $18^{\circ} \mathrm{C}$ for 5 hours, fixed in aceto-ethanol $(1: 3 \mathrm{v} / \mathrm{v})$ solution for 2 to 24 hours, and then stored in $70 \%$ ethanol until use. The $\mathrm{xBrassicoraphanus} \mathrm{flower} \mathrm{buds} \mathrm{were} \mathrm{fixed} \mathrm{in} \mathrm{the} \mathrm{same}$ solution for 24 hours and stored at $-20^{\circ} \mathrm{C}$ in $70 \%$ ethanol.

\section{Preparation of Somatic Metaphase Chromosome Spreads}

A modified version of the method described by Kato et al. (2004) was used to prepare chromosome spreads. Briefly, fixed root tips were thoroughly washed with distilled water, after which the meristem section of the roots was cut out and digested in 2\% cellulase (MB Cell, Korea), 1.5\% macerozyme (Maxim Bio, USA) and 1\% pectolyase (Sigma, Japan) in $150 \mathrm{mM}$ citrate buffer, $\mathrm{pH} 4.5$ for 90 minutes at $37^{\circ} \mathrm{C}$. The meristems were then thoroughly washed in ice-cold distilled water, after which the root epidermises were removed and the remaining section containing the dividing cells was pipetted into a tube with chilled acetoethanol $(1: 3 \mathrm{v} / \mathrm{v})$ solution, then suspended by gentle vortexing for 30 seconds. The cells were subsequently collected in the bottom of the tube by centrifugation and re-suspended in aceto- ethanol (9:1 v/v) solution. Finally, the cell suspension was dropped on pre-cleaned glass slides, placed in a humid chamber $\left(37^{\circ} \mathrm{C}\right)$ and slowly dried.

\section{Probe Preparation}

The procedure described by Lim et al. (2005) was used to obtain the 5S rDNA sequences of genomic DNA from B. rapa L. ssp. pekinensis and the fragment was labelled with dig-11-dUTP (Roche, Germany) by nick translation. The 45S rDNA sequence, containing a 9-kb fragment of 18S-5.8S-25SrDNA genes from wheat (Gerlach and Bedbrook, 1979) was isolated and labelled with biotin-16-dUTP (Roche, Germany) by nick translation. The $200-500$ bp labelled DNA fragments were used as probes.

For GISH analysis, total genomic DNA was extracted and purified from young leaves of B. rapa L. ssp. pekinensis 
and $R$. sativus L. using the CTAB- extraction method (Allen et al., 2006). The genomic DNA from B. rapa was labelled with dig-11-dUTP (Roche, Germany) by nick translation and used as a probe, while the unlabelled DNA of $R$ sativus was fragmented at $100^{\circ} \mathrm{C}$ for 7 minutes and used as a block. The lengths of the probe and the blocking DNA fragments were determined by agarose-gel electrophoresis within the range of 200-500 bp, after which samples were stored at $-20^{\circ} \mathrm{C}$ until use.

\section{Fluorescence in Situ Hybridization (FISH) and Genomic in Situ Hybridization (GISH)}

The FISH procedure described by Lim et al. (2005) and Waminal et al. (2012) was used in this study, with some modifications. Briefly, slides were pretreated with RNase A buffer $\left(100 \mu \mathrm{g} \cdot \mathrm{mL}^{-1}\right.$ RNase A in $2 x$ SSC $)$ at $37^{\circ} \mathrm{C}$ for 1 hour, after which they were soaked in $0.01 \mathrm{M} \mathrm{HCl}$ for 2 minutes. Next, samples were treated with $10 \%(\mathrm{w} / \mathrm{v})$ pepsin in a $1: 100$ dilution of $0.01 \mathrm{M} \mathrm{HCl}$ for 10 minutes at $37^{\circ} \mathrm{C}$ and then washed for 5 minutes with $2 x$ SSC. The slides were subsequently post-fixed with $4 \%$ paraformaldehyde in 2x SSC, washed with 2x SSC, and dehydrated in ethanol with a series of different concentrations. A hybridization mixture that consisted of $50 \%$ formamide, $10 \%$ dextransulfate, 2x SSC, $200 \mathrm{ng}$ salmon sperm DNA or $5 \mathrm{ng} \cdot \mu^{-1}$ unlabelled genomic DNA for GISH, and $50 \mathrm{ng} \cdot \mu \mathrm{L}^{-1}$ of each DNA probe (5S rDNA and 45S rDNA for FISH, or labelled genomic DNA for GISH) was prepared and then adjusted to $40 \mu \mathrm{L}$ per slide with nuclease free water. The FISH/GISH mixture was denatured at $90^{\circ} \mathrm{C}$ and cooled on ice for another 10 minutes, then mounted on slides and denatured at $80^{\circ} \mathrm{C}$ for 2.5 minutes, after which it was placed in an incubator at $37^{\circ} \mathrm{C}$ overnight (to 16 hours). The next day, the slides were washed in 2x SSC for 30 minutes at RT, 0.1x SSC for 35 minutes at RT and finally in 2x SSC for 15 minutes at $42^{\circ} \mathrm{C}$. Dig- labelled and biotin- labelled probes were detected with anti-dig-FITC conjugate (Sigma, USA) and streptavidin-Cy3 conjugate antibodies (Zymed Lab., USA), respectively. The antibodies were reacted on slides at $37^{\circ} \mathrm{C}$ for 1 hour, then washed in the detection buffer at $37^{\circ} \mathrm{C}$. Next, the slides were dehydrated in ethanol series $(70$, 90, and 100\%, 3 minutes each) and air-dried. A $40 \mu \mathrm{L}$ aliquot of 1:100 DAPI [f.c. $1 \mu \mathrm{g} \cdot \mathrm{mL}^{-1}$ (Roche, Germany)] in Vectashield (Vector Lab., Inc., USA) reagent was mounted to counterstain the chromosomes and covered with a glass coverslip.

\section{Meiotic Chromosome Pairing Analysis}

Meiotic chromosomes were prepared according to Zhong et al. (1996) and Ge and Li (2007) with minor modifications. Young flower buds from 1.6 to $2.0 \mathrm{~mm}$ and anthers from 0.8 to $1.0 \mathrm{~mm}$ long were used. Fixed flower buds were washed with distilled water for at least 10 minutes. One anther from a flower bud was squashed in $60 \%$ acetic acid, air-dried, stained with 4',6-diamidino-2-phenylindole (DAPI), and analyzed using a fluorescence microscope. Microsporocytes in prophase I were selected, and the remaining anthers were then treated with an enzyme mixture of $2 \%$ cellulase (MB Cell, Korea), 1\% cytohelicase (Yakult, Japan), and 1\% pectolyase (Sigma, Japan) for 90 minutes at $37^{\circ} \mathrm{C}$. Next, the treated anthers were washed in distilled water for 5 minutes, transferred to a glass slide, then squashed after adding 60\% acetic acid and air-dried. Meiotic chromosomes entering late prophase I were scored and meiotic pairing configurations and frequency were analysed using 100 pollen mother cells (PMCs) from different flower buds.

\section{Imaging and Karyotype Analysis}

The FISH images of each chromosome were measured three times to obtain the mean values. Homologous chromosomes were confirmed based on chromosome length, morphology and, FISH signal patterns. The chromosome arrangements of B. rapa and $R$. sativus were conducted according to the rDNA distribution patterns, according to the methods described by Lim et al. (2005) and Hwang et al. (2012), respectively. The overall chromosome size of $\mathrm{xBrassicoraphanus} \mathrm{line} \mathrm{BB \# 5} \mathrm{was} \mathrm{estimated} \mathrm{by} \mathrm{measuring}$ the lengths of short and long arms of chromosomes, excluding satellites and nucleolar organizing regions due to considerable variations in their size in each satellite chromosome and the chromosomes were arranged from longest to shortest. Images were captured using an Olympus BX-51 fluorescence microscope equipped with a chargecoupled device (CCD) camera (CoolSNAP ${ }^{\mathrm{TM}} \mathrm{cf}$ ) and analyzed using the Genus software (version 3.1). Final images were enhanced using Adobe Photoshop CS3. The method described by Levan et al. (1964) was used to classify the chromosome types. Homologous chromosomes were determined based on their lengths, morphological characteristics, and rDNAs signals.

\section{Results}

\section{Karyotype Analysis}

The mitotic chromosome number of Brassica rapa $\mathrm{L}$. ssp. pekinensis was $2 \mathrm{n}=20$, (Fig. 1A), with lengths ranging from 2.75 to $4.75 \mu \mathrm{m}$ and a total length of $36.22 \mu \mathrm{m}$ (Table 
1). The chromosome complement is comprised of eight metacentric and two submetacentric homologous chromosomes, respectively. Three and five pairs of $5 \mathrm{~S}$ and $45 \mathrm{~S}$ rDNA loci, respectively, were observed near the centromeric regions of B. rapa chromosomes. The 5S rDNA loci were observed on the terminal parts of the short arm of chromosome
\#2 and \#10 and near the centromeric part of the long arm of chromosome \#3 (Figs. 1A and 2). In the case of chromosomes \#2 and \#3, 5S rDNA was juxtaposed with $45 \mathrm{~S}$ rDNA on the short and long arms, respectively. The 45S rDNA loci of chromosomes \#1, \#3, \#4, and \#5 were observed near the centromeric regions of the long arms,

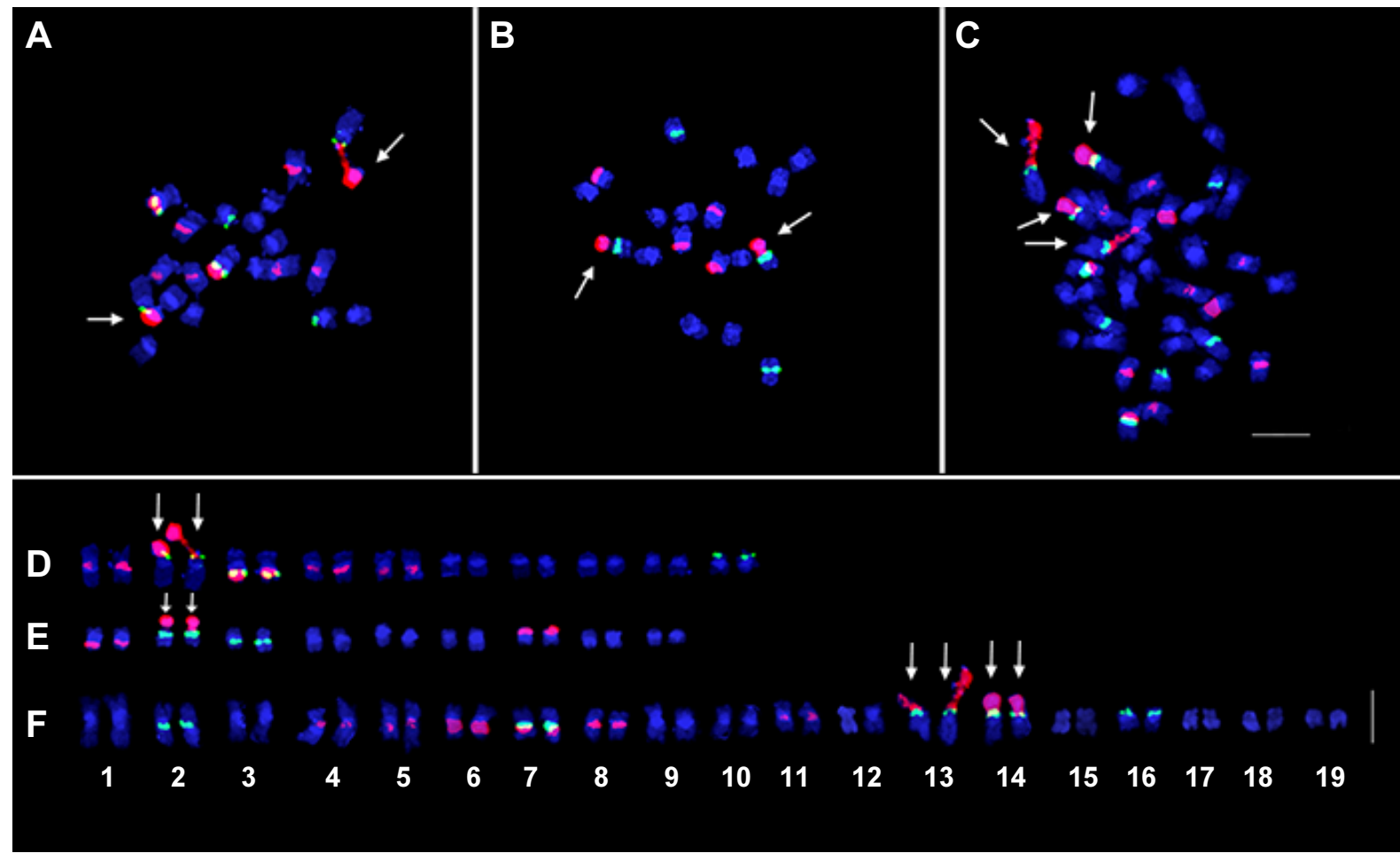

Fig. 1. FISH metaphases and their chromosome arrangement showing $5 \mathrm{~S}$ and $45 \mathrm{~S}$ rDNA signals in $B$. rapa L. ssp. pekinensis, $2 \mathrm{n}=20$ (A and D), $R$. sativus L. var. rafiphera, $2 \mathrm{n}=18(\mathrm{~B}$ and $\mathrm{E}$ ), and $\mathrm{xBrassicoraphanus} \mathrm{line} \mathrm{BB} \# 5,2 \mathrm{n}=38(\mathrm{C}$ and $\mathrm{F}$ ). The 5S and 45S rDNA loci are shown as green and red signals, respectively. The white arrows indicate the satellite chromosomes showing the nucleolar organizing region. Scale bar, $5 \mu \mathrm{m}$.

Table 1. Summary of FISH karyotype analyses of intergeneric hybrid xBrassicoraphanus line BB\#5 and its two parental lines, Brassica rapa L. ssp. pekinensis and Raphanus sativus L. var. rafiphera.

\begin{tabular}{|c|c|c|c|c|c|c|c|}
\hline \multirow{2}{*}{ Species } & \multirow{2}{*}{$\begin{array}{l}\text { Chr. no. } \\
\qquad(2 n)\end{array}$} & \multicolumn{3}{|c|}{ Chr. length $(\mu \mathrm{m})$} & \multicolumn{2}{|c|}{ rDNA signals } & \multirow{2}{*}{ Karyotype formula $(2 n)^{\mathrm{z}}$} \\
\hline & & Shortest & Longest & Total & $5 S$ & $45 S$ & \\
\hline B. rapa $\mathrm{L}$. & 20 & 2.75 & 4.76 & 36.22 & 3 & 5 & $\begin{array}{l}2 m+2 s m^{*}+2 m+2 m+2 m+2 m+2 s m+2 m+ \\
2 m+2 m\end{array}$ \\
\hline$R$ sativus $\mathrm{L}$. & 18 & 2.04 & 3.31 & 24.66 & 2 & 3 & $\begin{array}{l}2 m+2 s m^{*}+2 m+2 m+2 m+2 m+2 m+2 m+ \\
2 m\end{array}$ \\
\hline
\end{tabular}

${ }^{\mathrm{z}} \mathrm{m}$, metacentric; sm, submetacentric. The chromosomes in the karyotypic formula were arranged according to the chromosome number assigned in Fig. 2.

*Satellite chromosomes. 
while that of chromosome \#2, which showed the most intense signal, was on the satellite including the nucleolar organizing region (NOR). Previous studies reported three and five pairs of $5 \mathrm{~S}$ and $45 \mathrm{~S}$ rDNA loci in B. rapa (Hwang et al., 2009; Koo et al., 2011; Lim et al., 2005; Xiong and Pires, 2011;) and the same rDNA numbers and distribution patterns were observed in the present study.

The mitotic chromosome number of Raphanus sativus

Table 2. Frequency of meiotic chromosome pairing of the intergeneric hybrid xBrassicoraphanus line BB\#5.

\begin{tabular}{lc}
\hline Pairing pattern & PMCs (no.) \\
\hline 19 bivalent pairs & 42 \\
1 tetravalent and 17 bivalent pairs & 28 \\
2 tetravalent and 15 bivalent pairs & 24 \\
3 tetravalent and 13 bivalent pairs & 6 \\
Total & 100 \\
\hline
\end{tabular}

L. var. rafiphera was $2 \mathrm{n}=18$ (Fig. 1B), with lengths ranging from 2.04 to $3.31 \mu \mathrm{m}$ and a total length of $24.66 \mu \mathrm{m}$ (Table 2). The chromosome complement consisted of eight metacentric pairs and one submetacentric homologous chromosome pair. Two pairs of $5 \mathrm{~S}$ and three pairs of $45 \mathrm{~S}$ rDNA loci were observed in the $R$. sativus genome (Figs. 1B and 2). The 5S rDNA loci were observed on the terminal part of the short arm and near the centromeric part of the long arm of chromosomes \#2 and \#3, respectively. Two 45S rDNA loci were observed near the centromeric region of the long arm of chromosome \#1 and the intercalary region of the short arm of chromosome \#7. The largest 45S rDNA signal appears on the satellite segment that contains NOR on chromosome \#2 to which 5S rDNA is juxtaposed (Fig. 1B). These results coincided with those reported by Hwang et al. (2012).

The mitotic chromosome number of $\mathrm{xBrassicoraphanus}$ BB\#5 was $2 \mathrm{n}=38$ (Fig. 1C), with lengths ranging from 2.18 to $5.01 \mu \mathrm{m}$ and a total length of $65.68 \mu \mathrm{m}$ (Table 3).

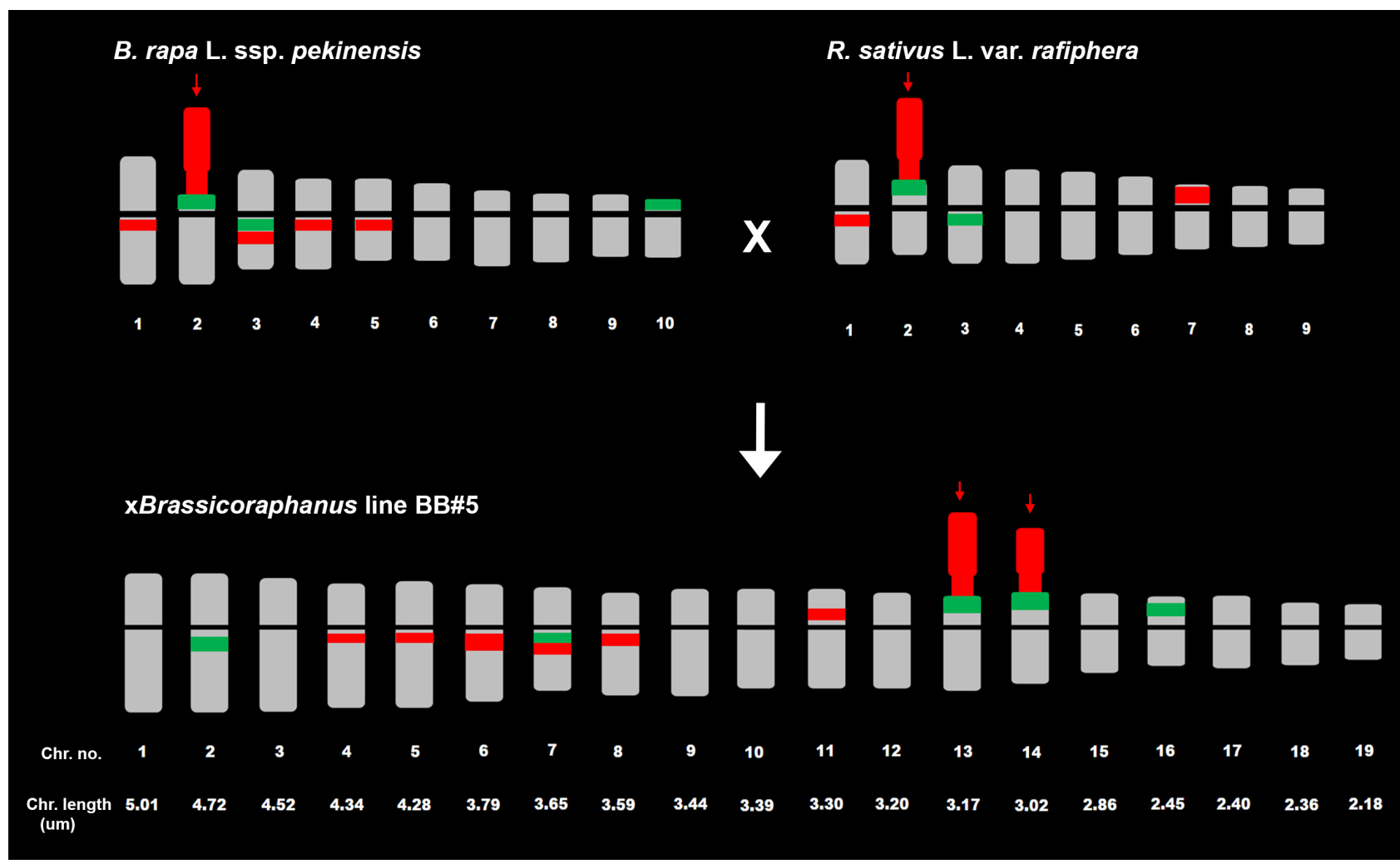

Fig. 2. FISH karyotype idiograms showing chromosome number, length, morphology, and 5S and 45S rDNA loci in $B$. rapa ssp. pekinensis, $R$ sativus L. and xBrassicoraphanus line BB\#5. The green color indicate the position of the 5S rDNA, while the red indicates 45S rDNA signals. The chromosome lengths are shown below the chromosome number. The chromosome arrangement of Brassica and Raphanus was conducted as described by Lim et al. (2005) and Hwang et al. (2012) according to the rDNA locus pattern and xBrassicoraphanus line BB\#5 based on chromosome lengths. Red arrows indicate satellite chromosomes. 
The chromosome complement is comprised of 17 metacentric and two submetacentric homologous chromosome pairs, respectively. There were five $5 \mathrm{~S}$ and eight $45 \mathrm{~S}$ rDNA loci observed on BB\#5 chromosomes (Figs. 1C and 3). The 5S rDNA loci were observed near the centromeric regions of the long arms of chromosomes \#2 and \#7, at the terminal parts of the short arms of chromosomes \#13 and \#14 while being juxtaposed to the NOR, and on the intercalary part of the short arm of chromosome \#16. The 45S rDNA loci were observed near the centromeric regions of the long arms of chromosomes \#4, \#5, \#6, \#7, and \#8, near that of the short arm of chromosome \#11, and on the satellite segment including NOR of chromosomes \#13 and \#14. According to the rDNA signal patterns, chromosome \#2 of BB\#5 was similar to chromosome \#3 of R. sativus; BB\#5 chromosomes \#4, \#5, \#6, and \#8 to chromosomes $\# 1$, \#4, and \#5 of B. rapa and chromosome \#1 of R. sativus; BB\#5 chromosome \#7 to chromosome \#3 of B. rapa; BB\#5 chromosome \#11 to chromosome \#7 of $R$. sativus; BB\#5 chromosome \#16 to chromosome \#10 of B. rapa; and BB\#5 satellite chromosomes \#13 and \#14 to satellite chromosome \#2 of B. rapa and $R$. sativus, respectively (Fig. 2).

Due to the small size and similar morphology of the chromosomes, accurate identification of the homologous chromosome using only DAPI staining without any chromosomespecific sequence markers was relatively difficult. Upon FISH karyotype analysis of $B$. rapa, $R$ sativus, and xBrassicaraphanus line $\mathrm{BB} \# 5$ using the $5 \mathrm{~S}$ and $45 \mathrm{~S}$ rDNA probes, the difficulties associated with identification of each chromosome were overcome by the presence, absence, position, or size of the rDNA signals. In hybrid line BB\#5, all rDNA signals from both the B. rapa and $R$ sativus genomes were detected, including two pairs of nucleolar organizing chromosomes that each resembled either that from the $B$ rapa or $R$. sativus genome. FISH karyotype analysis revealed that 10 of 19 chromosomes of the BB\#5 genome were identified as inherited chromosomes from each parental species.

When the FISH karyotype of BB\#5 was compared with that of each parental species, no apparent variation in chromosomal number or structure was detected at the microscopic level. Rather, complete sets of the two parental chromosomes appeared to reside in the same nucleus, strongly supporting that $\mathrm{xBrassicoraphanus} \mathrm{line} \mathrm{BB \# 5} \mathrm{is}$ a true allotetraploid derived from a cross between different genera, which is an extremely rare event in nature.

\section{Meiosis Pairing Analysis}

Although B. rapa and $R$. sativus belong to different genera, they are closely related species in the Brassicaceae family; accordingly, there must be significant homology between the two genomes. Recent genomic studies revealed that $B$. rapa and $R$ sativus share a high degree of synteny in their genomes (Kitashiba et al., 2014; Moghe and Shiu, 2014). This suggests the possibility that nonhomologous chromosome pairing can take place via homeologous regions during the early stages of meiotic prophase I, which may lead to the formation of multivalent chromosome pairs between chromosomes of $B$. rapa and $R$. sativus.

In the present meiotic pairing analysis at prophase I, 19 bivalent pairs were most frequently observed (Figs. $3 \mathrm{~A}$ and 3C), which accounted for $42 \%$ of the 100 PMCs examined (Table 2), whereas the rest consisted of a mixture of bivalent and tetravalent chromosomes. For example, $28 \%$ of the PMCs were found to have one tetravalent and 17 bivalents (Fig. 3D), while 24\% contained two tetravalents and 15 bivalents (Fig. 3E). Moreover, 6\% of the PMCs formed three tetravalents and 13 bivalents (Fig. 3F). These findings indicate that significant homology exists between $B$. rapa and $R$. sativus chromosomes and they are able to physically interact with each other to form multivalents during meiosis. Multivalent chromosome formation can generate various chromosomal changes via abnormal segregation in meiosis (Comai, 2005; Fujii and Ohmido, 2011; Leitch and Leitch, 2008). These findings suggest that there could be a high chance of chromosome rearrangement, such as translocation and deletion, when two nonhomologous chromosomes exchange segments, which conceivably leads to genome instability and infertility in newly synthesized allopolyploid plants. Despite the formation of multivalent chromosome pairs during meiosis, GISH analysis of BB\#5 clearly showed 10 bivalent chromosome pairs with green fluorescence signals, although the remaining chromosome pairs displayed no signals when $B$. rapa genomic DNA was used as a probe and $R$ sativus DNA as blocking DNA. These findings indicate that 10 pairs of chromosomes are B. rapaspecific and there is no massive chromosome rearrangement in $\mathrm{xBrasscoraphanus.}$

Poor fertility is always an obstacle that must be overcome in allopolyploid breeding (Prakash et al., 2009). However, the intergeneric hybrid line BB\#5 displays exceptionally high seed fertility and stable inheritance of morphological characters as demonstrated in another xBrassicoraphanus line (Lee et al., 2011). Overall, $42 \%$ of pollen mother cells analysed showed 19 complete bivalent chromosomes even though some appeared to have different numbers of tetravalents with different frequencies. These results strongly supports that stabilization of the intergeneric polyploid $\mathrm{xBrassicoraphanus} \mathrm{line} \mathrm{BB \# 5.}$ 

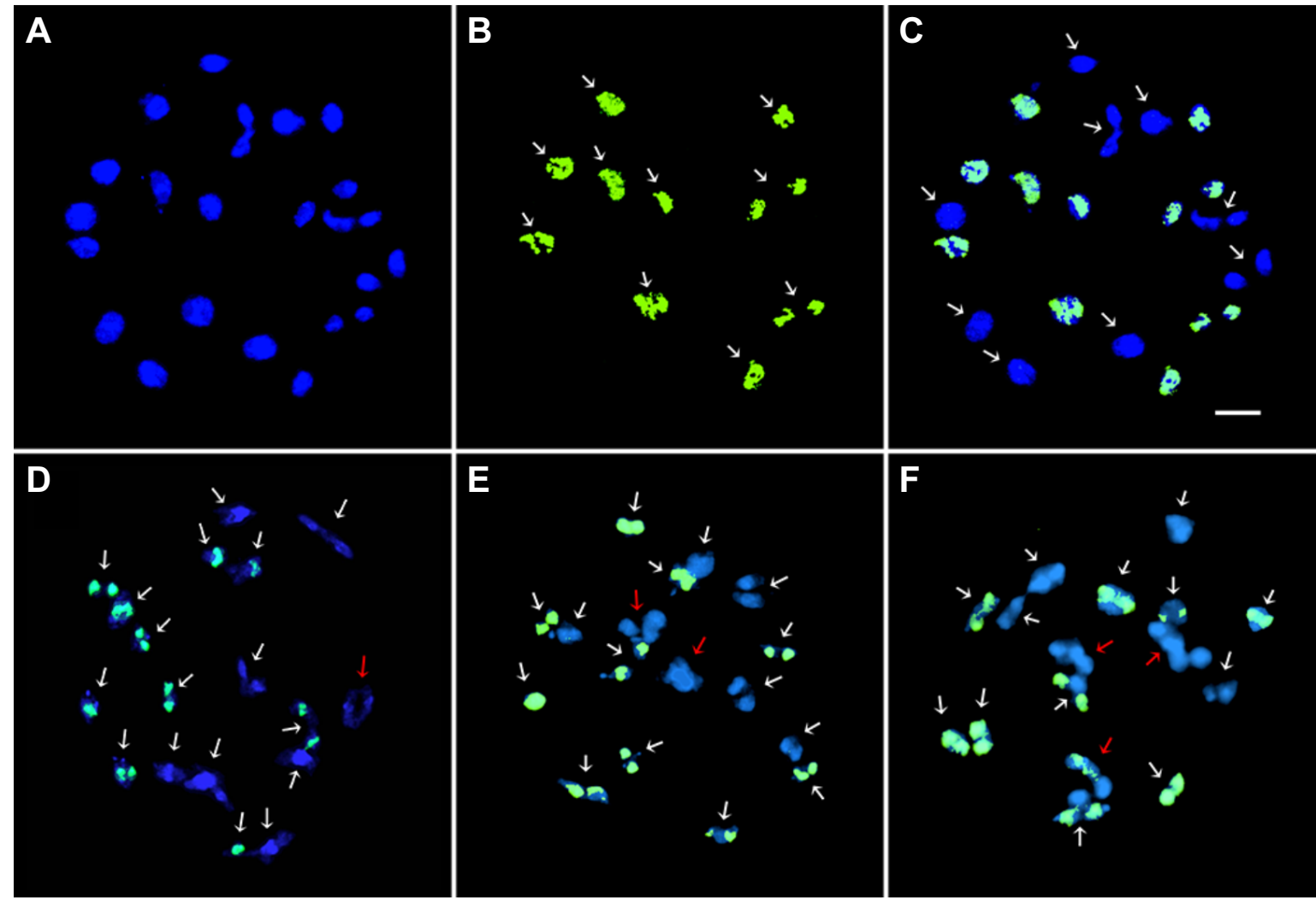

Fig. 3. GISH analyses of meiotic pairings in PMCs of xBrassicoraphanus line BB\#5. A, DAPI image (blue). B, GISH signals (green) of Brassica probe. C-F, merged images. Nineteen complete bivalents are shown (A), 10 of which are Brassica rapa chromosomes (arrows in B, green signals) and nine that are Raphanus sativus (arrows in C, blue). Meiotic chromosome compositions of one tetravalent and seventeen bivalents (D), two tetravalents and fifteen bivalents (E), and three tetravalents and thirteen bivalents (F) are also shown. In all cases, among 38 chromosome complements of xBrassicoraphanus line BB\#5, 10 pairs of Brassica rapa chromosomes were detected as green fluorescence. The white arrows in Figs. D, E, and F indicate bivalents, while red arrows indicate tetravalents. Scale bar, $5 \mu \mathrm{m}$.

\section{Discussion}

We analyzed the karyotype of somatic metaphase chromosomes by fluorescence in situ hybridization (FISH) and meiotic chromosome pairing of pollen mother cells (PMCs) by genomic in situ hybridization (GISH) in the newly synthesized allotetraploid xBrassicoraphanus line BB\#5.

Cytogenetic studies in natural and synthetic allopolyploids have been reported by many researchers (Bennett et al., 1992; Fujii and Ohmido, 2011; Yao et al., 2010). The FISH technique has been shown to be especially useful for more accurate karyotype analysis because it provides good diagnostic markers for identification of each chromosome. Tandem repeat sequences such as $5 \mathrm{~S}$ and $45 \mathrm{~S}$ rDNAs have primarily been used as probes for FISH (Fujii and Ohmido, 2011; Fukui et al., 1998; Hasterok et al., 2006; Kim et al., 1998; Lim et al., 2007; Snowdon et al., 1997). Several researchers applied FISH in Brassica rapa using 5S and 45S rDNA probes and reported three and five 5S and 45S rDNA pairs, respectively (Hwang et al., 2009; Koo et al., 2011; Lim et al., 2005; Xiong and Pires, 2011). Hwang et al. (2012) analyzed the FISH karyotype of Raphanus sativus and reported two and three 5S and 45S rDNA pairs, respectively. In our FISH karyotype analyses of Brassica rapa and Raphanus sativus using 5S and 45S rDNA probes, the same rDNA distribution patterns were observed and $\mathrm{xBrassicaraphanus}$ line BB\#5 appeared to have both combined rDNA patterns of two parental species. In addition, GISH was successful at identifying subgenomes of interspecific hybrid in Brassicaceae (Howell and Armstrong, 2013; Lim et al., 2012; Wang et al., 2006; Yao et al., 2010). Lim et al. (2012) applied GISH to discriminate each parental subgenomes on somatic metaphase chromosomes of the $\mathrm{xBrassicoraphanus} \mathrm{line} \mathrm{BB \# 4} \mathrm{and}$ confirmed 20 chromosomes of B. rapa in the hybrid genome. 
Here, we applied GISH to the meiotic prophase I cell of BB\#5. Ten pairs of Brassica bivalent chromosomes could clearly be discriminated in the intergeneric hybrid line.

Allopolyploids generally show genetic and phenotypic changes due to gene dosage effects, the existence of different genomes in a nucleus, variations in chromosome number, unbalanced parental genome contributions, and chromosomal rearrangements (Leitch and Leitch, 2008). Fujii and Ohmido (2011) observed several chromosome variations and aberrant meiosis in a synthetic hybrid and suggested that the genetic instability of the hybrid polyploid was due to abnormal chromosome number and structure caused by aberrant meiosis. In the present study, intergeneric allotetraploid $\mathrm{xBrassicoraphanus} \mathrm{line} \mathrm{BB \# 5} \mathrm{showed} \mathrm{very} \mathrm{stable} \mathrm{mitotic}$ chromosome constitution and meiotic pairing pattern, supporting the high seed fertility and morphological uniformity of BB\#5 even though we could not rule out the possibility of chromosomal rearrangement or structural changes in the next generations because of the appearance of different number of tetravalent with different frequencies according to the PMCs. However, such stable behaviour in somatic and meiotic cells is very rare in natural and synthetic intergeneric hybrids. Gaeta et al. (2007) observed that genomic changes in resynthesized Brassica napus were rare in the first generation, but that the changes were much more frequent in the fifth generation. Continuous cytogenetic and morphological study of BB\#5 should be conducted to confirm whether the chromosomal stability will be maintained during successive generations.

Consequently, a very stable xBrassicoraphanus line BB\#5 was newly developed by microspore mutation, which was certified by stable chromosome composition and meiotic pairing patterns in this study. Further molecular cytogenetic studies including FISH and GISH using more detailed chromosome-specific markers and comparative studies related to the genome sequencing are necessary to elucidate a more accurate genome structure of the hybrid line. The results presented herein will be useful for further genomic study of xBrassicoraphanus lines and their improvement as new promising breeding varieties.

\section{Literature Cited}

Allen, G.C., M.A. Flores-Vergara, S. Krasnyanski, S. Kumar, and W.F. Thompson. 2006. A modified protocol for rapid DNA isolation from plant tissues using cetyltrimethylammonium bromide. Nature Protocols 1:2320-2325.

Bennett, S.T., A.Y. Kenton, and M.D. Bennett. 1992. Genomic in situ hybridization reveals the allopolyploid nature of Milium montianum (Gramineae). Chromosoma 101:420-424.

Cao, W. 2003. Cytogenetic and molecular genetic evidence on evolution of genus Triticum, p. 223-247. In: A.K. Sharma and A. Sharma (eds.). Plant genome: Biodiversity and evolution. Phanerogams-Angiosperm. Science Publisher, Enfield, NH, USA.

Capdeville, G., M.T. Souza Junior, D. Szinay, L.E.C Diniz, E. Wijnker, R. Swennen, G.H.J. Kema, and H. Jong. 2008. The potential of high-resolution BAC-FISH in banana breeding. Euphytica 166:431-443. doi:10.1007/s10681-008-9830-2.

Comai, L. 2005. The advantages and disadvantages of being polyploidy. Nat. Rev. Genet. 6:836-846.

Devi, J., J.M. Ko, and B.B. Seo. 2005. FISH and GISH: Modern cytogenetic techniques. Indian J. Biotech. 4:307-315.

Dolstra, O. 1982. Synthesis and fertility of xBrassicoraphanus and ways of transferring Raphanus characters to Brassica. Agric. Res. Rep. 917:1-90.

Fujii, K. and N. Ohmido. 2011. Stable progeny production of the amphidiploid resynthesized Brassica napus cv. Hanakkori, a newly bred vegetable. Theor. Appl. Genet. 123:1433-1443.

Fukui, K., S. Nakayama, N. Ohmido, H. Yoshiaki, and M. Yamabe. 1998. Quantitative karyotyping of three diploid Brassica species by imaging methods and localization of $45 \mathrm{~S}$ rDNA loci on the identified chromosomes. Theor. Appl. Genet. 96:325-330.

Fukui, K. 2005. Recent development of image analysis method in plant chromosome research. Cytogene. Genome Res. 109(1-3): 83-89.

Gaeta, R.T., J.C. Pires, F. Iniguez-Luy, E. Leon, and T.C. Osborn. 2007. Genomic changes in resynthesized Brassica napus and their effect on gene expression and phenotype. Plant Cell 19:3403-3417.

Ge, X.H. and Z.Y. Li. 2007. Intra- and intergenomic homology of B-gemone chromosomes in trigenomic combinations of the cultivated Brassica species revealed by GISH analysis. Chromosome Res. 15:849-861. doi:10.1007/s10577-007-1168-4.

Gerlach, W.L. and J.R. Bedbrook. 1979. Cloning and characterization of ribosomal rDNA genes from wheat and barley. Nucleic Acids Res. 7:1869-1885.

Hasterok, R. and J. Maluszynska. 2000a. Cytogenetic markers of Brassica napus chromosomes. J. Appl. Genet. 41:1-9.

Hasterok, R. and J. Maluszynska. 2000b. Cytogenetic analysis of diploid Brassica species. Acta. Biol. Cracov. Ser. Bot. 42: 145-163.

Hasterok, R., E. Wolny, M. Hosiawa, M. Kowalczyk, S. KulakKsiazczyk, T. Ksiazczyk, W.K. Heneen, and J. Maluszyynska. 2006. Comparative analysis of rDNA distribution in chromosomes of various species of Brassicaceae. Ann. Bot. 97:205-216.

Howell, E.C. and S. Armstrong. 2013. Using sequential fluorescence and genomic in situ hybridization (FISH and GISH) to distinguish the $\mathrm{A}$ and $\mathrm{C}$ genomes in Brassica napus, p. 25-34. In: Plant meiosis: Methods and protocols, methods in molecular biology 990. Springer Science+Business Media, New York, USA. doi: 
10.1007/978-1-62703-333-6_3.

Hwang, Y.J., H.H. Kim, S.J. Kwon, T.J. Yang, H.C. Ko, B.S. Park, J.D. Chung, and K.B. Lim. 2009. Karyotype analysis of three Brassica species using five different repetitive DNA markers by fluorescence in situ hybridization. Kor. J. Hort. Sci. Technol. 27:456-463.

Hwang, Y.J., S.N. Lee, K.A. Song, K.B. Ryu, K.H. Ryu, and H.H. Kim. 2010. Karyotype analyses of genetically modified (GM) and non-GM hot peppers by conventional staining and FISH Method. Hort. Environ. Biotechnol. 51:525-530.

Hwang, Y.J., H.J. Yu, J.H. Mun, K.B. Ryu, B.S. Park, and K.B. Lim. 2012. Centromere repeat DNA originated from Brassica rapa is detected in the centromere region of Rapanus sativus chromosomes. Kor. J. Hort. Sci. Technol. 30:751-756.

Jellen, E.N., B.S. Gill, and T.S. Cox. 1994. Genomic in situ hybridization differentiates between $\mathrm{A} / \mathrm{D}$ and $\mathrm{C}$-genome chromatin and detects intergenomic translocations in polyploidy oats species (genus Avena). Genome 37:613-618.

Kato, M. and S. Tokumasu. 1983. The stability of chromosome numbers and the maintenance of euploidy in Brassicoraphanus. Euphytica 32:415:423.

Kato, A., J.C. Lamb, and J.A. Birchler. 2004. Chromosome painting using repetitive DNA sequences as probes for somatic chromosome identification in maize. Proc. Natl Acad. Sci.101: 13554-13559. doi:10.1073/pnas.0403659101.

Kenton, A., A.S. Parokonny, Y.Y. Gleba, and M.D. Bennett. 1993. Characterization of the Nicotiana tabacum L. genome by molecular cytogenetics. Mol. Gen. Genet. 240:159-169.

Kim, S.Y., Y.P. Lim, and J.W. Bang. 1998. Cytogenetic analysis of Brassica campestris var. pekinensis using C-banding and FISH. Genes Genom. 20:285-294.

Kitashiba, H., F. Li, H. Hirakawa, T. Kawanabe, Z. Zou, Y. Hasegawa, K. Tonosaki, S. Shirasawa, A. Fukushima, S. Yokoi, Y. Takahata, T. Kakizaki, M. Ishida, S. Kamoto, K. Sakamoto, K. Shirasawa, S. Tabata, and T. Nishio. 2014. Draft sequences of the radish (Raphamus sativus L.). Genome DNA Res. p. 1-10. doi:10.1093/dnares/dsu014.

Koo, D.H., C.P. Hong, J. Batley, Y.S. Chung, D. Edwards, J.W. Bang, Y. Hur, and Y.P. Lim. 2011. Rapid divergence of repetitive DNAs in Brassica relatives. Genomics 97:173-185.

Lee, S.S., H.D. Kim, D.K. Oh, and J.K. Woo. 1999. Breeding a fertile intergeneric allotetraploid plant between heading Chinese cabbage and Korean radish. Kor. J. Hort. Sci. Tech. 17:653-653.

Lee, S.S., W.J. Choi, and J.G. Woo. 2002. Development of new vegetable crop in xBrassicoraphanus by hybridization of Brassica campestris and Raphanus sativus. J. Kor. Soc. Hort. Sci. 43:693-698.

Lee, S.S., S.A. Lee, J.M. Yang, and J.K. Kim. 2011. Developing stable progenies of $x$ Brassicoraphanus, an intergeneric allopolyploid between Brassica rapa and Raphanus sativus, through induced mutation using microspore culture. Theor. Appl. Genet. 122:
885-891.

Leitch, A.R. and I.J. Leitch. 2008. Genomic plasticity and the diversity of polyploidy plants. Science 320:481-483.

Levan, A., K. Fredga, and A.A. Sandberg. 1964. Nomenclature for centromeric position on chromosomes. Hereditas 52:517-523.

Levsky, J.M. and R.H. Singer. 2003. Fluorescence in situ hybridization: Past, present and future. J. Cell Sci. 116:2833-2838.

Lim, K.B., J.H. De Jong, T.J. Yang, J.Y. Park, S.J. Kwon, J.S. Kim, M.H. Lim, J.A. Kim, M.A. Jin, S.H. Kim, Y.P. Lim, J.W. Bang, H.I. Kim, and B.S. Park. 2005. Characterization of rDNA and tandem repeats in the heterochromatin of Brassica rapa. Mol. Cell. 19:436-444.

Lim, K.B., T.J. Yang, Y.J. Hwang, J.S. Kim, J.Y. Park, S.J. Kwon, J.A. Kim, B.S. Choi, M.H. Lim, M. Jin, H. de Jong, I. Bancroft, Y.P. Lim, and B.S. Park. 2007. Characterization of the centromere and per-centromere retrotransposons in Brassica rapa and their distribution in related Brassica species. Plant J. 49:173-183.

Lim, S.J., S.S. Lee, and J.W. Bang. 2012. Karyotype and genomic in situ hybridization pattern in $x$ Brassicoraphamus, an intergeneric hybrid between Brassica campestris ssp. pekinensis and Raphamus sativus. Pl. Biotech. Rep. 6:107-112.

Lou, Q., M. Iovene, D.M. Spooner, C. Robin Buell, and J.M. Jiang. 2010. Evolution of chromosome 6 of Solanum species revealed by comparative fluorescence in situ hybridization mapping. Chromosoma 119:435-442.

Maluszynska, J. and J.S. Heslop-Harrison. 1993. Physical mapping of rDNA loci in Brassica species. Genome 36:774-781.

McNaughton, I.H. 1979. The current position and problems in the breeding of Raphanubrassica (radicole) as a forage crop. Proc. $4^{\text {th }}$ Eucarpia-Conf. Breed Cruciferous Crops p. 22-28.

Moghe, G.D. and S.H. Shiu. 2014. The causes and molecular consequences of polyploidy in flowering plants. Ann. New York Acad. Sci. 1320:16-34.

Namai, H., M. Sarashima, T. Hosoda. 1980. Interspecific and intergeneric hybridization breeding in Japan, p. 191-204. In: S. Tsunoda, K. Hinata, and C. Gomez-campo (eds.) Brassica crop and wild allies. Japan. Sci. Soc. Tokyo.

Olsson, G. and S. Ellerstrom. 1980. Polyploidy breeding in Europe, p. 167-190. In: S. Tsunoda, K. Hinata, and C. Gómez-Campo (eds.). Brassica crops and wild allies. Japan. Sci. Soc. Tokyo.

Park, H.M., E.J. Jeon, N.E. Waminal, K.S. Shin, S.J. Kweon, B.S. Park, S.C. Suh, and H.H. Kim. 2010. Detection of transgenes in three genetically modified rice lines by fluorescence in situ hybridization. Genes Genomics 32:527-531. doi:10.1007/s13258010-0064-z.

Prakash, S., S.R. Bhat, C.F. Quiros, P.B. Kirti, and V.L. Chopra. 2009. Brassica and its close allies: cytogenetics and evolution, p. 21-187. In: J. Jules (ed.). Plant breed reviews, Vol. 31. John Wiley and Sons. Inc. London.

Snowdon, R.J., W. Köhler, and A. Köhler. 1997. Chromosomal localization of rDNA loci in the Brassica A and C genomes. Genome 40:582-587. 
Tokumasu, S. 1976. The increase of seed fertility of Brassicoraphamus through cytological irregularity. Euphytica 25:463-470.

Vasconcelos, S., A.A. Souza, C.L. Gusmao, M. Milani, A.M. Benko-Iseppon, and A.C. Brasileiro-Vidal. 2010. Heterochromatin and $5 \mathrm{~S}$ and $45 \mathrm{~S}$ rDNA sites as reliable cytogenetic markers for castor bean (Ricinus communis, Euphorbiaceae). Micron 41:746-753. doi:10.1016/j.micron.2010.06.002.

Waminal, N.E., H.M. Park, K.B. Ryu, J.B. Kim, T.J. Yang, and H.H. Kim. 2012. Karyotype analysis of Panax ginseng C.A. Meyer, 1843 (Araliaceae) based on rDNA loci and DAPI band distribution. Comp. Cytogen. 6:425-441.

Yang, Q., L. Hanson, M.D. Bennett, and I.L. Leitch. 1999. Genome structure and evolution in the allohexaploid weed Avena fatua L. (Poaceae). Genome 45:512-518.

Yao, X.C., X.H. Ge, J.P. Chen, and Z.Y. Li. 2010. Intra- and intergenomic relationships in interspecific hybrids between Brassica (B. rapa, B. napus) and a wild species B. maurorum as revealed by genomic in situ hybridization (GISH). Euphytica 173:113-120.

Wang, Y., K. Sonntag., E. Rudloff, P. Wehling, and R.J. Snowdon. 2006. GISH analysis of disomic Brassica napus-Crambe abyssinica chromosome addition lines produced by microspore culture from monosomic addition lines. Plant Cell Rep. 25:35-40.

Xiong, Z.Y. and J.C. Pires. 2011. Karyotype and identification of all homoeologous chromosomes of allopolyploid Brassica napus and its diploid progenitors. Genetics 187:37-49.

Zhong, X.B., J. Hans de Jong, and P. Zabel. 1996. Preparation of tomato meiotic pachytene and mitotic metaphase chromosomes suitable for fluorescence in situ hybridization (FISH). Chromosome Res. 4:24-28. doi:10.1007/BF02254940. 